\title{
Screening Phosphorylation Site Mutations in Yeast Acetyl-CoA Carboxylase Using Malonyl-CoA Sensor to Improve Malonyl-CoA-Derived Product
}

\author{
Xiaoxu Chen ${ }^{1+}$, Xiaoyu Yang ${ }^{1+}$, Yu Shen ${ }^{1}$, Jin Hou ${ }^{1 *}$ and Xiaoming Bao ${ }^{1,2 *}$ \\ 'State Key Laboratory of Microbial Technology, School of Life Science, Shandong University, Jinan, China, ${ }^{2}$ Shandong \\ Provincial Key Laboratory of Microbial Engineering, Qilu University of Technology, Jinan, China
}

OPEN ACCESS

Edited by:

Xueyang Feng,

Virginia Tech, United States

Reviewed by:

Xiaochao Xiong,

Washington State University,

United States

Sun Xinxiao,

Beijing University of Chemical

Technology, China

${ }^{*}$ Correspondence:

$\mathrm{Jin} \mathrm{Hou}$

houjin@sdu.edu.cn

Xiaoming Bao

bxm@sdu.edu.cn

tThese authors are joint first authors.

Specialty section:

This article was submitted to

Microbial Physiology and Metabolism,

a section of the journal

Frontiers in Microbiology

Received: 10 November 2017 Accepted: 09 January 2018

Published: 25 January 2018

Citation:

Chen $X$, Yang $X$, Shen $Y$, Hou J and

Bao $X$ (2018) Screening

Phosphorylation Site Mutations in Yeast Acetyl-COA Carboxylase

Using Malonyl-CoA Sensor

to Improve Malonyl-CoA-Derived

Product. Front. Microbiol. 9:47.

doi: 10.3389/fmicb.2018.00047
Malonyl-coenzyme A (malonyl-CoA) is a critical precursor for the biosynthesis of a variety of biochemicals. It is synthesized by the catalysis of acetyl-CoA carboxylase (Acc1p), which was demonstrated to be deactivated by the phosphorylation of Snf1 protein kinase in yeast. In this study, we designed a synthetic malonyl-CoA biosensor and used it to screen phosphorylation site mutations of Acc1p in Saccharomyces cerevisiae. Thirteen phosphorylation sites were mutated, and a combination of three site mutations in Acc1p, S686A, S659A, and S1157A, was found to increase malonyl-CoA availability.

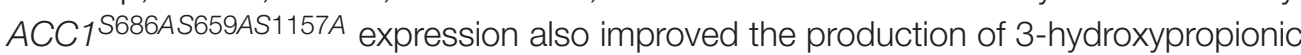
acid, a malonyl-CoA-derived chemical, compared to both wild type and the previously reported ACC1 ${ }^{S 659 A S 1157 A}$ mutation. This mutation will also be beneficial for other malonyl-CoA-derived products.

Keywords: malonyl-CoA sensor, phosphorylation site mutations, acetyl-CoA carboxylase, 3-hydroxypropionic acid, Saccharomyces cerevisiae

\section{INTRODUCTION}

Saccharomyces cerevisiae is a very potential microbial cell factory for production of a wide range of fuels and chemicals due to its robustness and high tolerance to environmental stresses. When engineering the cell factory to produce these products, the precursor availability is one of key limiting factors that affects the product titer. Malonyl-coenzyme A (malonyl-CoA) is a major precursor for the production of many important biochemicals and biofuels, such as polyketides, flavonoids, and fatty acid-derived chemicals (Dixon and Steele, 1999; Hara et al., 2006; Christian, 2009; Chooi and Tang, 2013). However, the intracellular level of malonyl-CoA is very low because malonyl-CoA synthesis is tightly regulated in S. cerevisiae (Li et al., 2014). Therefore, insufficient malonyl-CoA synthesis largely limits the production of malonyl-CoA-derived products.

In $S$. cerevisiae, cytosolic malonyl-CoA is synthesized from acetyl-CoA by acetyl-CoA carboxylase (Acc1) and it is subsequently used for fatty acid synthesis. Acclp is crucial for fatty acids biosynthesis and the maintenance of the nuclear envelope. Acetyl-CoA carboxylases are large, multidomain enzymes and contain a biotin carboxylase (BC) domain, a carboxyltransferase (CT) domain and a biotin carboxyl carrier protein (BCCP) domain. Eukaryotic ACCs also contain two non-catalytic regions, the large central domain $(\mathrm{CD})$ and the $\mathrm{BC}-\mathrm{CT}$ interaction domain $(\mathrm{BT})$ 
(Hunkeler et al., 2016). As the rate-limiting enzyme for fatty acid metabolism, Acclp is tightly regulated both transcriptionally and post-translationally. Similar to other phospholipid synthesis pathway genes, the transcription of ACC1 is regulated by the positive transcription factors Ino2p and Ino4p and the negative regulator Opilp (Hasslacher et al., 1993). The enzyme activity of Acclp is also negatively regulated by AMP-activated protein kinase (Snf1) in S. cerevisiae (Woods et al., 1994). It is reported that the phosphorylation of Acclp by Snflp inactivates the catalytic ability of Acclp. The activation of Snflp is triggered during growth under glucose derepression conditions (less than $0.05 \%$ glucose) (Woods et al., 1994), then the activated Snflp phosphorylates Acclp at one or more phosphorylation sites and results in the partial deactivation of Acclp. However, potential residues for Acclp phosphorylation are not fully understood. Feng et al. (2015) reported that deletion of Snf1 did not increase the 1-hexadecanol production, but overexpressing $A C C 1$ in $\triangle S N F 1$ strain increased the production by $50 \%$ (Feng et al., 2015). Asides from regulating $A C C 1$, Snf1 is also a global regulator for carbon metabolism, $\beta$-oxidation, and stress response (Zhang et al., 2011). Thus, SNF1 deletion may not specifically increase malonyl-CoA derived products.

Choi and Da Silva (2014) aligned the Rattus norvegicus (rat) Acclp with yeast Acclp and identified an amino acid (Ser1157) that is critical for phosphorylation and causing deactivation of the enzyme. The introduction of a S1157A mutation in Acclp increased its activity and enhanced polyketide 6-methylsalicylic acid and native fatty acid production (Choi and Da Silva, 2014). A recent report demonstrated that the phosphorylation of the Ser1157 residue by Snflp triggered a conformational change, leading to a disassociated $\mathrm{BC}$ domain dimer, thus inactivating the catalytic ability of Acclp (Wei et al., 2016). Shi et al. identified two phosphorylation sites that were regulated by Snflp by comparing the phosphorylation recognition motif (Hyd-X-Arg-XX-Ser-XXX-Hyd) (Dale et al., 1995) and introduced a two-site mutation (S659A and S1157A) in Acclp. These mutations enhanced the activity of Acclp and subsequently increased the production of two malonyl-CoA-derived products, fatty acid ethyl esters and 3-hydroxypropionic acid (3-HP) (Shi et al., 2014). However, although these studies reported the positive role of mutations S659A and S1157A, it is still uncertain whether Snflp regulates Acclp only by phosphorylating these two sites. Li et al. (2014) manually examined the modification sites on Acclp using MIDAS and confirmed that 15 sites were phosphorylated. The authors also noted that both the native state and phosphorylated state were identified at the same time, indicating that the regulation of phosphorylation is complicated.

The Acc catalytic activity determination is complicated, therefore limited the high-throughput screening the mutation libraries of the enzyme. Intracellular malonyl-CoA concentration is another way to reflect the Acc activity. The malonylCoA biosensor has been designed in S. cerevisiae to facilitate the malonyl-CoA determination using a bacterial transcription factor FapR (fatty acid and phospholipid biosynthesis regulator) and its DNA binding site (fapO operator) (Schujman et al., 2003). Li et al. (2015) designed a malonyl-CoA sensor by inserting a fapO operator immediately upstream the TATA box in GPM1 promoter and expression of an adapted FapR to monitor the malonyl-CoA levels in S. cerevisiae. By using this sensor to search a genome-wide overexpression library, two genes, TPI1 and PMP1, were identified that improved the intracellular malonyl-CoA concentration, thereby improving 3-HP production (Li et al., 2015). David introduced three fapO operators in yeast TEF1 promoter to increase the sensitivity of responding the malonyl-CoA concentration and used it to modulate pathway flux. The introduction of dual pathway control increased 3-HP production (David et al., 2016). However, these sensors either have a relatively high leaking transcription or low dynamic change response to malonyl-CoA levels (Table 1).

In this study, we aimed to construct a malonyl-CoA sensor in S. cerevisiae that has higher sensitivity and low leaky transcription which is very important for library screening. The sensor we designed showed better response to malonyl-CoA concentrations than previously reported sensors. Then we used the sensor to detect the effect of mutating all possible phosphorylation sites of Acclp. A mutation, Acclp (S686AS659AS1157A), that could increase the concentration of malonyl-CoA and the production of 3-HP (a chemical derived from malonyl-CoA) (Figure 1), was isolated.

\section{MATERIALS AND METHODS}

\section{Medium and Growth Conditions}

Saccharomyces cerevisiae strain CEN.PK102-5B (MATa; ura3-52; his $3 \Delta 1$; leu2-3,112) was used as the background strain. Synthetic complete (SC) dropout medium was used for recombinant yeast strain selection. The SC medium contained $1.7 \mathrm{~g} / \mathrm{L}$ yeast nitrogen base (BBI Life Science Corporation, China), 5 g/L ammonium sulfate, complete supplement mixture (without uracil, histidine, or leucine) (Sunrise Science Products, United States), and $20 \mathrm{~g} / \mathrm{L}$ glucose. In total, $800 \mu \mathrm{g} / \mathrm{mL}$ G418 (Promega Corporation, United States) was used for selecting strains with the $\mathrm{Kan}^{r}$ marker. For routine cloning procedures, Escherichia coli Trans2Blue (Beijing TransGen Biotech Co., Ltd, China) was used and grown in Luria-Bertani (LB) medium with $100 \mu \mathrm{g} / \mathrm{L}$ ampicillin.

\section{Plasmid and Strain Construction}

All the plasmids and strains used in this study are listed in Supplementary Tables S1 and S2. The primers used in this study are listed in Supplementary Table S3. Yeast strain transformation was accomplished using the LiAc method. The obtained plasmids were verified by DNA sequencing.

The malonyl-CoA reductase encoding gene MCR from Chloroflexus aurantiacus was codon optimized, digested with NotI and SacI, and ligated into the $2 \mu$ plasmid pIYC04 under the control of the TEF1 promoter and ADH1 terminator. T4 DNA ligase (New England Biolabs, United States) was used for plasmid construction. 
TABLE 1 | Designs and characters of the malonyl-CoA sensors in S. cerevisiae.

\begin{tabular}{|c|c|c|c|c|c|}
\hline $\begin{array}{l}\text { Promoter used } \\
\text { in sensor }\end{array}$ & $\begin{array}{l}\text { Numbers of } \\
\text { FapO operator }\end{array}$ & $\begin{array}{l}\text { Level of } \\
\text { FapR }\end{array}$ & $\begin{array}{l}\text { The fold change of } \\
\text { fluorescence intensity } \\
\text { (with FapR/without FapR) }\end{array}$ & $\begin{array}{l}\text { The fold change of fluorescence } \\
\text { intensity (with saturated } \\
\text { cerulenin/without cerulenin) }\end{array}$ & Reference \\
\hline GPM1 & 1 & High & $29.8 \%$ & 4 & Li et al., 2015 \\
\hline TEF1 & 3 & Low & $14 \%$ & 1.9 & David et al., 2016 \\
\hline $\begin{array}{l}\text { TEF1 UAS and } \\
\text { GAL } 1 \text { core }\end{array}$ & 1 & High & $0.05 \%$ & 8 & This study \\
\hline
\end{tabular}

For a high level of $f a p R$ expression, the codon-optimized $f a p R$ was digested with SacI and SalI and then ligated into the pre-digested $2 \mu$ plasmid pYX242WS, resulting in the plasmid pYX242WS-fapR. A synthetic promoter was constructed by combining $203 \mathrm{bp}$ of the TEF1 promoter upstream activation sequence and $147 \mathrm{bp}$ of the GAL1 core promoter (with an identified TATA box). The FapR binding site, $17 \mathrm{bp}$ of the fapO operator (TTAGTATCAGGTACTAA), was inserted into the GAL1 core promoter, $7 \mathrm{bp}$ immediately behind the TATA box and replaced the original sequence to obtain the synthetic promoter GALlp(7)fapO (Figure 2A) (Schujman et al., 2003; Blazeck et al., 2012; Xu et al., 2014). The yeGFP (yeast-enhanced green fluorescent proteinencoding gene) was fused with the synthetic promoter by fusion PCR, and the fragment was then digested with Aat II and EcoRI and inserted into the pre-digested pYX242WS or pYX242WS-fapR plasmid to create pJfapO and pJfapOfapR. To obtain synthetic promoters GAL1p(61)fapO and fapO(0)GAL1p, the fapO operator was inserted $61 \mathrm{bp}$ behind or immediately upstream the TATA box separately. Each of the synthetic promoters was fused with yeGFP and then was cloned into a $2 \mu$ plasmid to obtain plasmid $\operatorname{pIFapO}(0)$ and pIFapO(61) through yeast homologous recombination. The codon-optimized fapR with SV40 NLS sequence at the $3^{\prime}$ end was digested with NotI and SacI and then cloned into plasmids $\mathrm{pIFapO}(0)$ and $\mathrm{pIFapO}(61)$ separately to create pIFapO(0)-FapR and pIFapO(61)-FapR. To obtain a low level of $f a p R$ expression, $f a p R$, which was controlled by the TEF1 promoter, was integrated into the yeast genome using $\mathrm{Kan}^{r}$ as the selection marker. The plasmid pJFE3-fapO was transformed into the fapR integration strain. The sequence information of fapR and $M C R$ was included in supplemental data.

Site-directed mutagenesis was introduced by designing mutant sites containing primers. A full ACC1 sequence with mutated residues was obtained by fusion PCR. ACC1 mutations were inserted into the $2 \mu$ plasmid pJFE3 under the control of the TEF1 promoter and PGK1 terminator through yeast homologous recombination. The constructed plasmids were verified by PCR and sequencing.

\section{Malonyl-CoA Sensor Activity and Acc1p Mutation Assay}

Fluorescence intensity was used to evaluate the sensor activity. The sensor-carrying strains were pre-cultured overnight. The overnight culture was collected and inoculated into $3 \mathrm{~mL}$ of

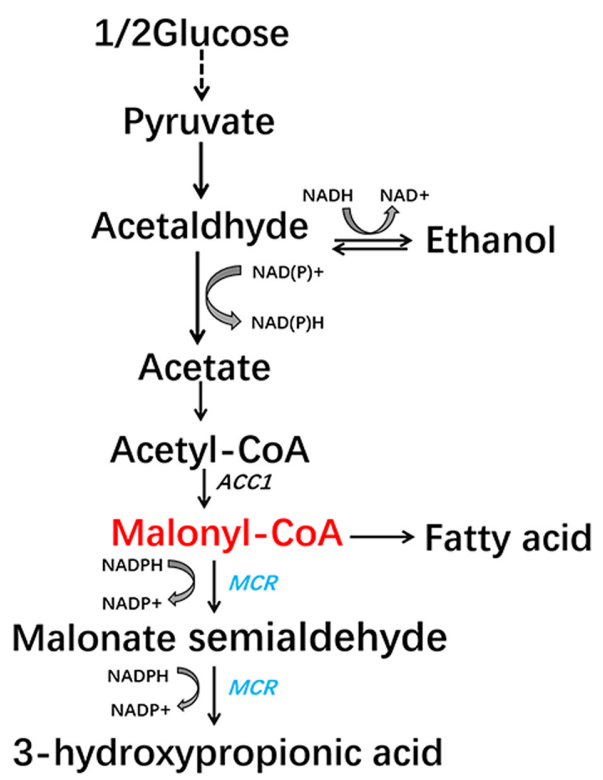

FIGURE 1 | Malonyl-CoA biosynthesis metabolic pathway and the engineered 3-hydroxypropionic acid synthesis pathway in S. cerevisiae.

fresh SC medium at an initial OD600 of 1 at $30^{\circ} \mathrm{C}, 200 \mathrm{rpm}$, and different concentrations of cerulenin $(0,0.5,2.5,5,10$, $15 \mu \mathrm{M})$ were added if necessary. For the fluorescence intensity assay, a $1 \mathrm{~mL}$ culture was isolated after $2 \mathrm{~h}$ of growth and centrifuged at $4^{\circ} \mathrm{C}$ and $13,000 \mathrm{rpm}$ for $2 \mathrm{~min}$, and the pellets were washed twice with distilled water. A $200 \mu \mathrm{L}$ cell suspension was used for fluorescence intensity measurement in triplicate. Fluorescence was measured using a 1420 Multilabel Counter (VICTOR ${ }^{3} \mathrm{~V}$, PerkinElmer, United States). The excitation and emission wavelengths for GFP were $485 \pm 20$ and $585 \pm 20$, respectively. The cell density was measured at $600 \mathrm{~nm}$ (Eppendorf BioPhotometer, Germany). The fluorescence intensity (a.u.) was determined relative to cell density. To detect the fluorescence intensity of the mutations, the strains were pre-cultured overnight, inoculated in shake flasks at an initial OD600 of 0.2 in $10 \mathrm{~mL}$ of SC medium, and cultivated $30^{\circ} \mathrm{C}$ at $200 \mathrm{rpm}$. Samples were taken after $6 \mathrm{~h}$, and fluorescence was measured as described above.

\section{3-HP Fermentation}

For 3-HP fermentation, strains were pre-cultured in $5 \mathrm{~mL}$ of SC dropout medium, and the seed culture was transferred into a 
A
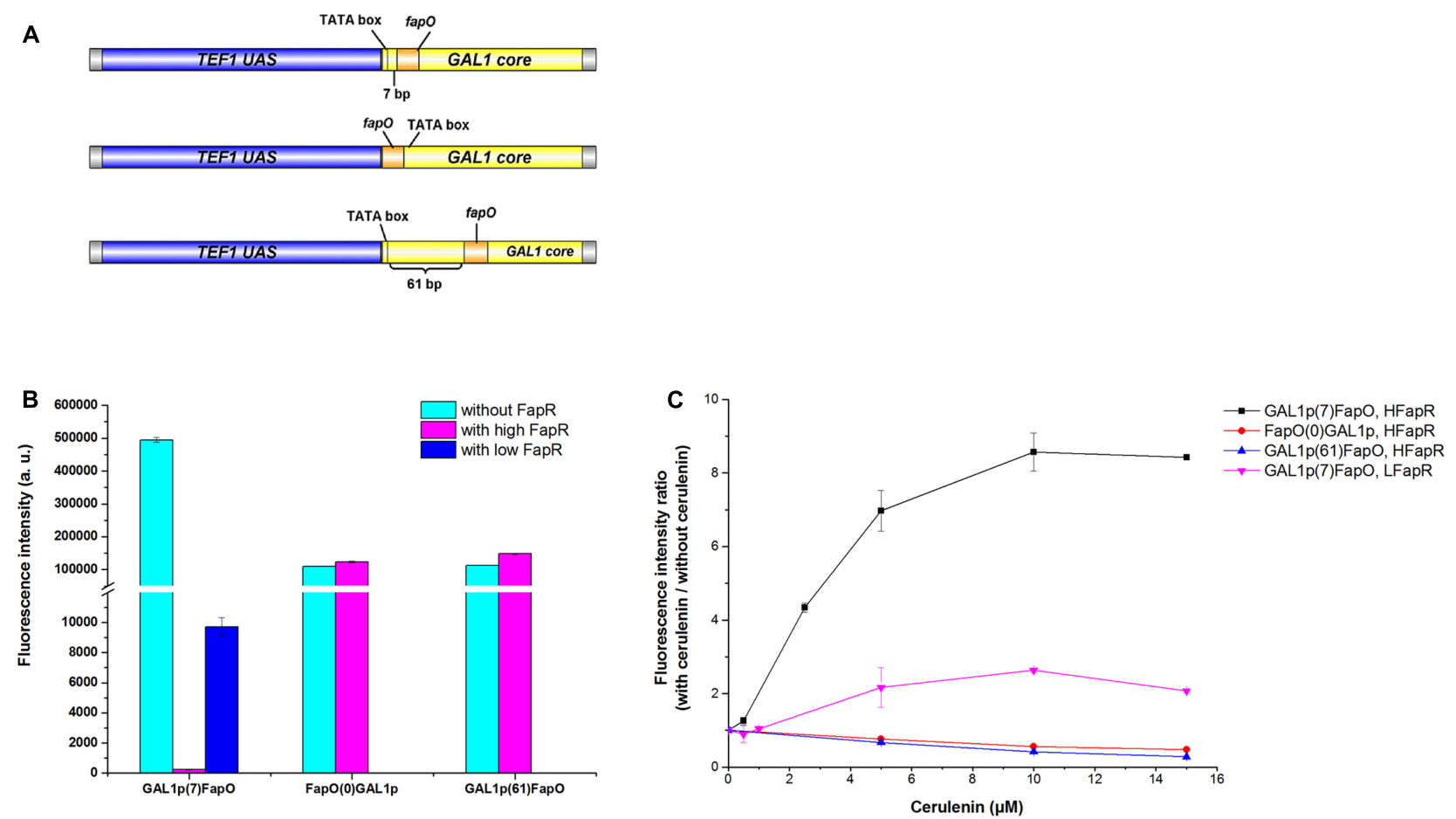

FIGURE 2 | Design and characters of the synthetic malonyl-CoA sensors. (A) Design of the synthetic promoters. The positions of fapO sequence were as follows: 7 bp downstream the TATA box of GAL1 core promoter, immediately upstream the TATA box and 61 bp downstream the TATA box of GAL 1 core promoter. (B) Comparing of the fluorescence intensity of the sensors between with FapR and without FapR. The synthetic promoters were cloned in multiple-copy plasmid. Fluorescence intensity (a.u.) means total fluorescence normalized by cell density (OD600). (C) The fluorescence intensity fold change of the sensors with gradient concentration of cerulenin addition relative to zero cerulenin addition. HFapR: fapR was expressed in a multicopy plasmid; LFapR: fapR was integrated into genome.

$100 \mathrm{~mL}$ shake flask at a working volume of $40 \mathrm{~mL}$. The initial OD600 of the culture was 0.2 . Fermentation was performed at $200 \mathrm{rpm}$ and $30^{\circ} \mathrm{C}$. Samples were taken every $12 \mathrm{~h}$ until $72 \mathrm{~h}$. 3-HP was analyzed by HPLC (Shimadzu Corporation, Japan) equipped with an Aminex HPX-87H column (Bio-Rad, Hercules, United States) at $65^{\circ} \mathrm{C}$ using $2.5 \mathrm{mM} \mathrm{H}_{2} \mathrm{SO}_{4}$ as the mobile phase at a flow rate of $0.6 \mathrm{~mL} / \mathrm{min}$.

\section{RESULTS}

\section{Design an Efficient Malonyl-CoA Sensor in S. cerevisiae}

Malonyl-CoA acts as a direct and specific inducer of FapRregulated promoters in Bacillus subtilis (Schujman et al., 2006). FapR binds to fapO (the operator region on the fap regulon) in the promoter region, which blocks the access of RNA polymerase II, thus repressing the transcription of downstream fatty acid pathway genes. Malonyl-CoA interacts with FapR and triggers a conformational change in FapR, consequently enabling FapR to disassociate from DNA and relieve the transcription repression. Based on that, we designed a malonyl-CoA sensor in $S$. cerevisiae and used $y e G F P$ as a reporter to monitor the intracellular malonyl-CoA levels. To obtain a more efficient sensor, the leaking transcription should be low, which means that when fapR binding to fapO, the basic transcription level should be low. In addition, the sensor also needs to response to the change of malonyl-CoA level sensitively, which means that the dynamic change of the fluorescence intensity should be high. Therefore, we chose a synthetic hybrid promoter containing the upstream activated sequence (UAS) of TEF1 promoter and core promoter sequence (begin with TATA box) of GAL1. The UAS from TEF1 allowed the high expression of downstream gene without fapR binding, and TATA box in the core promoter facilitate to decide the locations of fapO operator. The FapR binding site, $17 \mathrm{bp}$ of the fapO operator, was inserted either $7 \mathrm{bp}$ or 61 bp downstream of the TATA box in GAL1 core promoter, or immediately upstream the TATA box, and yeGFP was controlled by these synthetic hybrid promoters in a $2 \mu$ plasmid (Figure 2A). The codon-optimized $f a p R$ was expressed under the control of the TEF1 promoter in a $2 \mu$ plasmid. When fapO was inserted $7 \mathrm{bp}$ downstream of the TATA box [GAL1p(7)fapO for briefness], the transcription was almost completely shut down. The fluorescence intensity with FapR was only $0.05 \%$ relative to that without FapR, demonstrating the efficient repression effect of FapR when binding to this position (Figure 2B). However, when fapO was inserted 61 bp downstream of the TATA box [GAL1p(61)fapO for briefness] or immediately upstream of the TATA box [fapO(0)GAL1p for briefness], no transcription repression was observed, demonstrating that the location of $f a p O$ 
on these two sites was not efficient enough for FapR to inhibit transcription initiation.

Besides the effect of $f a p O$ location in promoter, we also detected the expression level of FapR to the behavior of the sensor. The fapR was integrated into the genome to obtain low expression level, and the yeGFP was controlled by the GAL1p(7)fapO synthetic promoter. We found that compared with the FapR expressed in a $2 \mu$ plasmid, a low level of FapR expression resulted in high fluorescence intensity which implicated that low FapR expression caused high leaky transcription (Figure 2B).

Cerulenin inhibits fatty acid synthesis by specific inhibition of $\beta$-ketoacyl-acyl carrier protein synthetase (Kawaguchi et al., 1982), and inhibits malonyl-CoA consumption for fatty acid synthesis. Thus, the intracellular malonyl-CoA concentration correlates with the amount of cerulenin added to the culture. As shown in Figure 2C, as for the sensor containing the GALlp(7)fapO synthetic promoter, with a gradient supply of cerulenin, the fluorescence intensity also increased gradually, reaching to eightfold with $10 \mu \mathrm{M}$ cerulenin (saturated concentration) relative to the condition without cerulenin in high FapR expression strain. The fluorescence intensity only increased onefold with cerulenin supply in low FapR expression strain (Figure 2C). As expected, the fluorescence intensity of the sensor containing fapO(0)GAL1p or GAL1p(61)fapO synthetic promoter did not increase with increased dose of cerulenin addition, indicating these two designs could not respond the malonyl-CoA concentration. These results demonstrated that high FapR expression combined with GAL1p(7)fapO synthetic promoter showed better response to the change of malonyl-CoA levels.

Comparing with the previously reported malonyl-CoA sensor (Table 1), the sensor with GAL1p(7)fapO synthetic promoter and high FapR expression designed in this study showed a low leaky transcription and a better sensitivity to the change of malonylCoA levels, which are very important properties for library screening.

\section{Site-Directed Mutations of Phosphorylation Sites of Acc1p Increased Intracellular Malonyl-CoA Levels}

Although mutations of Ser659 and Ser1157 phosphorylation sites have been shown to improve the synthesis of malonylCoA-derived products, it remains unclear whether other phosphorylation sites may have a positive impact. In this work, we mutated 13 predicted phosphorylation sites of Acclp according to Li et al.'s (2014) report (Figure 3A), which confirmed 15 phosphorylation sites of Acclp via MIDAS. The other two predicted phosphorylation residues are not existed in the Acclp from CEN.PK background strain used in this work. The phosphorylation residues were mutated to alanine. The sensor plasmid was then transformed into the Acclp mutation-containing strains to screen the malonyl-CoAoverproducing mutants. We found that the fluorescence intensity of strains containing $\operatorname{Acclp}(\mathrm{S} 686 \mathrm{~A})$ and $\operatorname{Acclp}(\mathrm{T} 1823 \mathrm{~A})$ was increased relative to the wild type, even though the increased range was still lower than strain with the previously reported Acclp(S659AS1157A) double mutation. The remaining phosphorylation site mutations did not show a positive effect on malonyl-CoA levels (Figure 3B). We further combined S686A and T1823A single mutations with the S659AS1157A double mutation to see if the combination mutations can achieve further increase in malonyl-CoA generation. As shown in Figure 3C, the introduction of the S686A mutation further improved the fluorescence intensity of the ACC1 ${ }^{S 659 A S 1157 A}$ expressing strain, whereas T1823A did not further enhance the fluorescence intensity, although the fluorescence intensity of the Acclp(T1823A) mutant was higher than wild-type Acclp. These results indicated that Acclp(S686AS659AS1157A) can produce more malonyl-CoA and should have higher enzyme activity.

\section{The Effect of Site Mutations of Acc1p to 3-HP Production}

We then analyzed the production of 3-HP, a malonyl-CoAderived chemical, to determine whether the increase in malonylCoA would result in higher production of malonyl-CoAderived chemical (Figure 1). The heterogeneous gene $M C R$, which encodes a bifunctional enzyme that includes malonylCoA reductase and 3-hydroxypropionate dehydrogenase, was overexpressed in Acclp mutation strains (Hugler et al., 2002). The titer of 3 -HP in the $A C C 1^{S 659 A S 1157 A}$-expressing strain was improved by 1.2 -fold relative to the wild type, which is consistent with the positive role of the S659AS1157A mutation in a previous study (Shi et al., 2014). The titer of 3-HP in the ACC1 $1^{S 686 A S 659 A S 1157 A}$-expressing strain was improved by 1.5-fold compared with the wild type, which is higher than the ACC1 ${ }^{\text {S659AS1157A }}$-expressing strain (Figure 4). This finding confirmed that the S686AS659AS1157A mutation of Acclp contributed to an increase in malonyl-CoA availability. However, the S686A single mutation of Acclp did not improve 3-HP production. This result indicated that S686A could benefit 3HP production only when integrated with the S659AS1157A mutation. Additionally, although the fluorescence intensity of $A C C 1^{T 1823 A}$ - and $A C C 1^{T 1823 A S 659 A S 1157 A}$-expressing strains was higher than the wild type, 3-HP production did not increase, excluding the positive role of T1823A for 3-HP generation. Cell growth of the engineered strains was not obviously changed by ACC1 mutation expression (Supplementary Figure S2).

\section{DISCUSSION}

The low production of malonyl-CoA in yeast constrains the biosynthesis of many valuable malonyl-CoA-derived compounds, such as flavonoids, polyketides, and fatty acid-derived fuels. Malonyl-CoA is generated from acetyl-CoA by the catalysis of acetyl-CoA carboxylase. The activity of Acclp is tightly regulated, and the phosphorylation of Acclp by Snf1 protein kinase can inactivate Acclp. Mutations of the phosphorylation sites Ser1157 and Ser659 have been shown to enhance the activity of Acclp and consequently the ability to synthesize malonyl-CoA-derived 
A
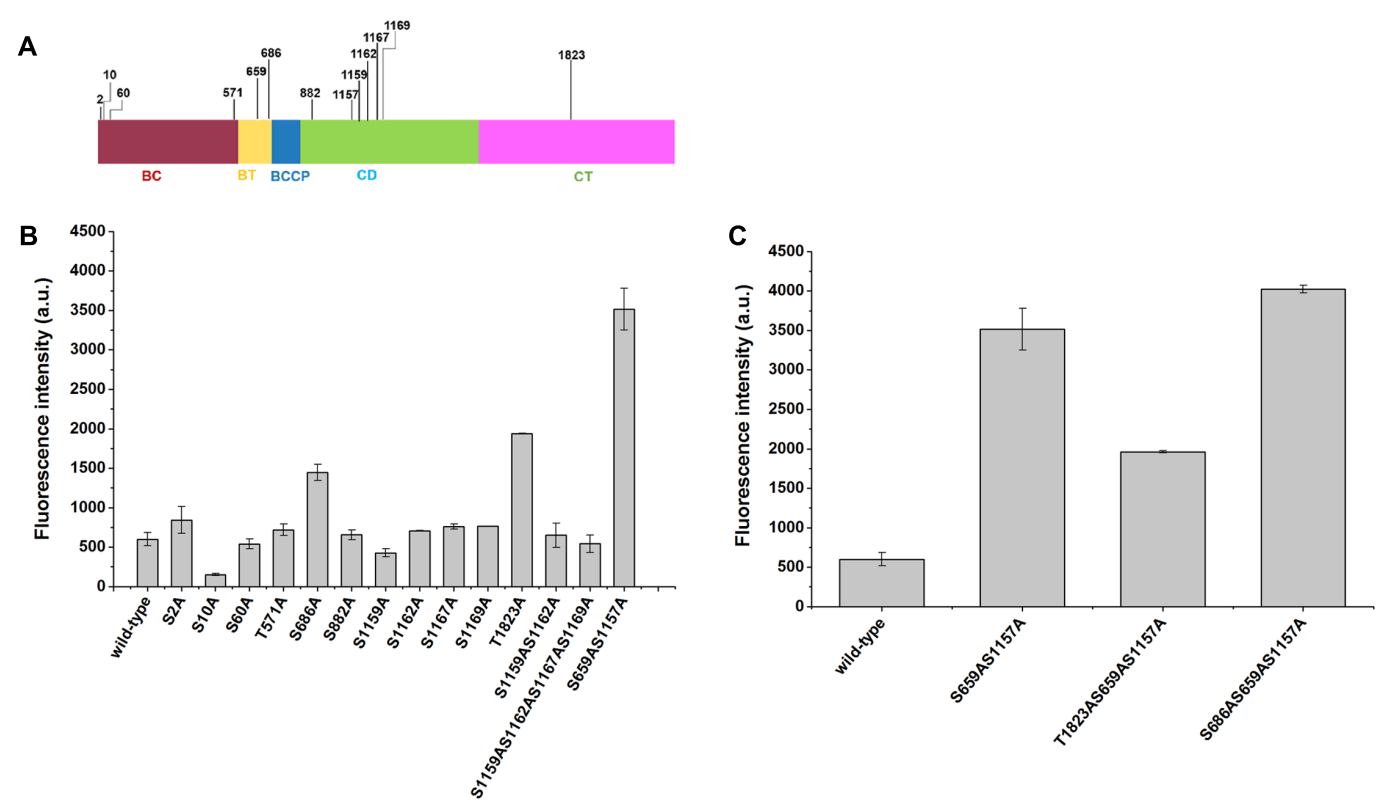

FIGURE 3 | Positions of the phosphorylation sites in Acc1p and the fluorescence intensity of phosphorylation mutation strains containing the malonyl-CoA sensor. (A) Position of the 13 phosphorylation sites in our study. (B) Fluorescence intensity at $6 \mathrm{~h}$ of the strains. Wild-type: CEN.PK102-5B with an empty plasmid and malonyl-CoA sensor plasmid. (C) Fluorescence intensity of the strains with combined mutations. The fluorescence intensity was measured at exponential phase.

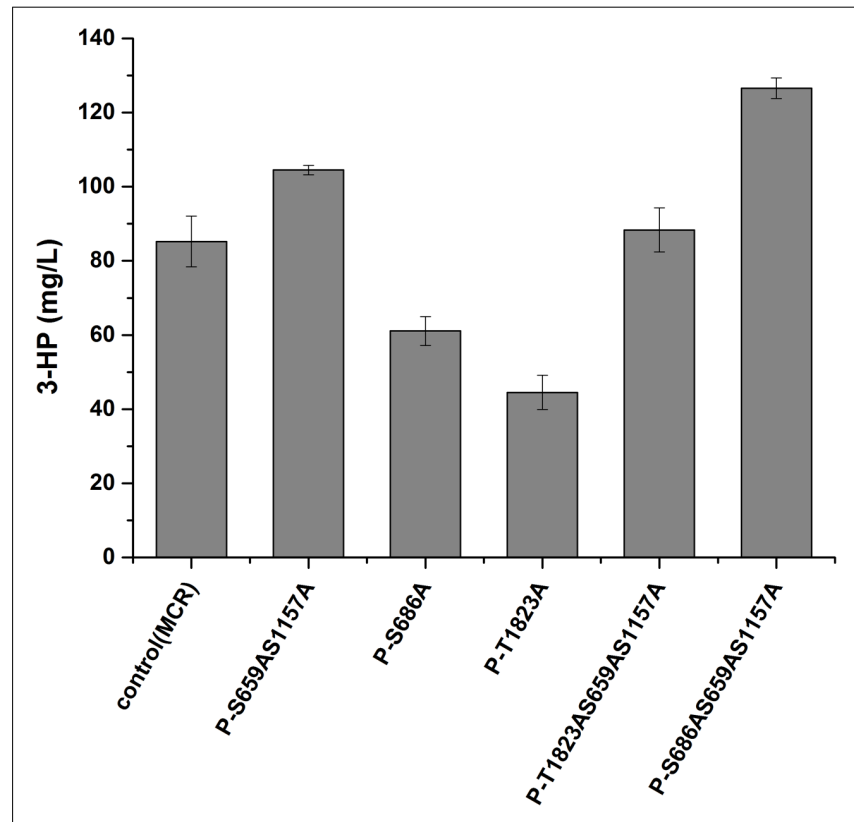

FIGURE 4 | 3-HP production by the strains expressing phosphorylation site mutations of Acc1p. Samples were taken at $72 \mathrm{~h}$ in shake flask fermentation. Control (MCR): CEN.PK102-5B with the MCR-containing plasmid.

products (Choi and Da Silva, 2014). However, there are more phosphorylation sites in Acclp. Li et al. (2014) predicted 15 possible phosphorylation sites in Acclp by in vitro experiments, and it is necessary to explore the role of these phosphorylation sites in the enzymatic bioactivity of acetyl-CoA carboxylase.
To facilitate the screening of phosphorylation site mutations, a malonyl-CoA sensor was constructed. Compared with the other two previously reported malonyl-CoA sensors ( $\mathrm{Li}$ et al., 2015; David et al., 2016), the sensor we designed in this work showed low leaky transcription and better sensitivity to the change of malonyl-CoA concentrations (Table 1). Although these malonyl-CoA sensors used operators from the same origin ( $f a p O$ from $B$. subtilis), the behavior is very different when inserting the operator to different promoters. As for the TEF1-GAL1 synthetic promoter in this work, the transcription was not repressed when fapO was located immediately upstream TATA box, while the same position in GPM1 promoter worked well. When the distance between fapO and the TATA box in TEF1GAL1 synthetic promoter extended to $61 \mathrm{bp}$, the transcription repression effect was disappeared. It seems that inserting one operator $7 \mathrm{bp}$ downstream of TATA box in TATA-containing promoter is enough for FapR to repress the transcription initiation, whereas it is not the case when the operators were inserted to the TATA-less promoter TEF1 where three operators were needed to repress the transcription effectively. In fact, the promoter property and operator locations are important to decide the performance of the biosensors.

Among the phosphorylation site mutations, the S659AS1157A double mutation increased 3-HP production, which confirmed the role of these phosphorylation sites. Combining the S686A mutation with the S659AS1157A double mutation further increased 3-HP production, suggesting that the phosphorylation site mutation of Ser686 further improved the enzyme activity of Acclp. Ser1157 phosphorylation triggers a conformational change that disables the formation of the BC dimer domain, thus eliminating the catalysis activity (Hunkeler et al., 2016). However, 
there is no direct evidence regarding the inhibitory regulation induced by the phosphorylation of Ser659. By analyzing the Acclp structure (from RCSB Protein Data Bank) using PyMOL software, we speculated that phosphorylation of Ser659 might influence the biotin moiety binding by regulating the active loop in BC domain. It is possible that the phosphorylation of Ser686 affected the enzyme activity through indirectly regulating the covalent state of Ser659 (Supplementary Figure S1).

We found that the fluorescence intensity was not completely consistent with 3-HP production. Only the strains with much higher fluorescence intensity showed a beneficial effect on 3HP production. It is probably because malonyl-CoA is an intermediate metabolite and can be consumed easily. Synthetic malonyl-CoA sensor can show the transient malonyl-CoA concentration. However, the production of 3-HP revealed the amount of malonyl-CoA flux to the downstream products. Therefore, when using a malonyl-CoA sensor to do highthroughput screening, it is essential to set up a fluorescence intensity threshold to obtain the mutations with higher products titer.

In this work, we studied the impact of phosphorylation regulation on the activity of acetyl-CoA carboxylase. We discovered that a combination mutation with S686A, S659A, and S1157A could benefit 3-HP production better than the previously reported S659S1157A mutation. Moreover, the malonyl-CoA sensor designed in this work was demonstrated to be a useful tool to screen mutations with improved malonyl-CoA pools.

\section{REFERENCES}

Blazeck, J., Garg, R., Reed, B., and Alper, H. S. (2012). Controlling promoter strength and regulation in Saccharomyces cerevisiae using synthetic hybrid promoters. Biotechnol. Bioeng. 109, 2884-2895. doi: 10.1002/bit. 24552

Choi, J. W., and Da Silva, N. A. (2014). Improving polyketide and fatty acid synthesis by engineering of the yeast acetyl-CoA carboxylase. J. Biotechnol. 187, 56-59. doi: 10.1016/j.jbiotec.2014.07.430

Chooi, Y. H., and Tang, Y. (2013). Navigating the fungal polyketide chemical space: from genes to molecules. ChemInform 44, 9933-9953. doi: 10.1021/jo30 $1592 \mathrm{k}$

Christian, H. (2009). The biosynthetic logic of polyketide diversity. Angew. Chem. Int. Ed. Engl. 48, 4688-4716. doi: 10.1002/anie.200806121

Dale, S., Wilson, W. A., Edelman, A. M., and Hardie, D. G. (1995). Similar substrate recognition motifs for mammalian AMP-activated protein kinase, higher plant HMG-CoA reductase kinase-A, yeast SNF1, and mammalian calmodulin-dependent protein kinase I. FEBS Lett. 361, 191-195. doi: 10.1016/ 0014-5793(95)00172-6

David, F., Nielsen, J., and Siewers, V. (2016). Flux control at the malonyl-CoA node through hierarchical dynamic pathway regulation in Saccharomyces cerevisiae. ACS Synth. Biol. 5, 224-233. doi: 10.1021/acssynbio.5b00161

Dixon, R. A., and Steele, C. L. (1999). Flavonoids and isoflavonoids - a gold mine for metabolic engineering. Trends Plant Sci. 4, 394-400. doi: 10.1016/S13601385(99)01471-5

Feng, X., Lian, J., and Zhao, H. (2015). Metabolic engineering of Saccharomyces cerevisiae to improve 1-hexadecanol production. Metab. Eng. 27, 10-19. doi: 10.1016/j.ymben.2014.10.001

Hara, T., Sato, T., Oka, M., Mori, S., and Shirai, H. (2006). Microdiesel: Escherichia coli engineered for fuel production. Microbiology 152, 2529-2536. doi: 10.1099/ mic.0.29028-0

Hasslacher, M., Ivessa, A. S., Paltauf, F., and Kohlwein, S. D. (1993). Acetyl-CoA carboxylase from yeast is an essential enzyme and is regulated by factors that control phospholipid metabolism. J. Biol. Chem. 268, 10946-10952.

\section{AUTHOR CONTRIBUTIONS}

$\mathrm{XB}$ and $\mathrm{JH}$ designed the experiments. $\mathrm{XC}$ and $\mathrm{XY}$ carried out the experiments. YS analyzed the experimental results.

\section{FUNDING}

This work was supported by the National Natural Science Foundation of China (31770101, 31470163, and 31711530155), and the Key R\&D Program of Shandong Province (2017GSF21110 and 2015GSF121015).

\section{ACKNOWLEDGMENTS}

We thank Caiyun Sun from Analysis and Testing Center of SKLMT (State Key Laboratory of Microbial Technology, Shandong University) for assistance in liquid chromatography and microplate reader.

\section{SUPPLEMENTARY MATERIAL}

The Supplementary Material for this article can be found online at: https://www.frontiersin.org/articles/10.3389/fmicb. 2018.00047/full\#supplementary-material

Hugler, M., Menendez, C., Schagger, H., and Fuchs, G. (2002). Malonylcoenzyme a reductase from Chloroflexus aurantiacus, a key enzyme of the 3-hydroxypropionate cycle for autotrophic $\mathrm{CO}_{2}$ fixation. J. Bacteriol. 184, 2404-2410. doi: 10.1128/JB.184.9.2404-2410.2002

Hunkeler, M., Stuttfeld, E., Hagmann, A., Imseng, S., and Maier, T. (2016). The dynamic organization of fungal acetyl-CoA carboxylase. Nat. Commun. 7:11196. doi: 10.1038/ncomms11196

Kawaguchi, A., Tomoda, H., Nozoe, S., Omura, S., and Okuda, S. (1982). Mechanism of action of cerulenin on fatty acid synthetase. Effect of cerulenin on iodoacetamide-induced malonyl-CoA decarboxylase activity. J. Biochem. 92, 7-12. doi: 10.1093/oxfordjournals.jbchem.a133933

Li, S., Tong, S., Wang, M., and Zhao, H. (2015). Development of a synthetic malonyl-CoA sensor in Saccharomyces cerevisiae for intracellular metabolite monitoring and genetic screening. Acs Synth. Biol. 4, 1308-1315. doi: 10.1021/ acssynbio.5b00069

Li, X., Guo, D., Cheng, Y., Zhu, F., Deng, Z., and Liu, T. (2014). Overproduction of fatty acids in engineered Saccharomyces cerevisiae. Biotechnol. Bioeng. 111, 1841-1852. doi: 10.1002/bit.25239

Schujman, G. E., Guerin, M., Buschiazzo, A., Schaeffer, F., Llarrull, L. I., Reh, G., et al. (2006). Structural basis of lipid biosynthesis regulation in Gram-positive bacteria. EMBO J. 25, 4074-4083. doi: 10.1038/sj.emboj.76 01284

Schujman, G. E., Paoletti, L., Grossman, A. D., and De Mendoza, D. (2003). FapR, a bacterial transcription factor involved in global regulation of membrane lipid biosynthesis. Dev. Cell 4, 663-672. doi: 10.1016/S1534-5807(03) 00123-0

Shi, S., Chen, Y., Siewers, V., and Nielsen, J. (2014). Improving production of malonyl coenzyme A-derived metabolites by abolishing Snf1dependent regulation of Acc1. mBio 5:e1130-14. doi: 10.1128/mBio.011 30-14

Wei, J., Zhang, Y., Yu, T. Y., Sadre-Bazzaz, K., Rudolph, M. J., Amodeo, G. A., et al. (2016). A unified molecular mechanism for the regulation of acetyl-CoA carboxylase by phosphorylation. Cell Discov. 2:16044. doi: 10.1038/celldisc. 2016.44 
Woods, A., Munday, M. R., Scott, J., Yang, X., Carlson, M., and Carling, D. (1994). Yeast SNF1 is functionally related to mammalian AMP-activated protein kinase and regulates acetyl-CoA carboxylase in Vivo. J. Biol. Chem. 269, 19509-19515.

Xu, P., Wang, W., Li, L., Bhan, N., Zhang, F., and Koffas, M. A. (2014). Design and kinetic analysis of a hybrid promoter-regulator system for malonyl-CoA sensing in Escherichia coli. ACS Chem. Biol. 9, 451-458. doi: 10.1021/cb400623m

Zhang, J., Vaga, S., Chumnanpuen, P., Kumar, R., Vemuri, G. N., Aebersold, R., et al. (2011). Mapping the interaction of Snf1 with TORC1 in Saccharomyces cerevisiae. Mol. Syst. Biol. 7:545. doi: 10.1038/msb.2011.80
Conflict of Interest Statement: The authors declare that the research was conducted in the absence of any commercial or financial relationships that could be construed as a potential conflict of interest.

Copyright $\odot 2018$ Chen, Yang, Shen, Hou and Bao. This is an open-access article distributed under the terms of the Creative Commons Attribution License (CC BY). The use, distribution or reproduction in other forums is permitted, provided the original author(s) or licensor are credited and that the original publication in this journal is cited, in accordance with accepted academic practice. No use, distribution or reproduction is permitted which does not comply with these terms. 